\title{
MicroRNA-296 inhibits colorectal cancer cell growth and enhances apoptosis by targeting ARRB1-mediated AKT activation
}

\author{
ZHE ZHANG ${ }^{*}$, XIAOHUA ZHONG ${ }^{*}$, YIPIN XIAO and CHAO CHEN \\ Department of Gastrointestinal Surgery, Huizhou Municipal Central Hospital, Huizhou, Guangdong 516001, P.R. China
}

Received May 14, 2018; Accepted October 9, 2018

DOI: $10.3892 /$ or.2018.6806

\begin{abstract}
Colorectal cancer (CRC) is the most commonly diagnosed tumor worldwide. However, the molecular mechanisms and biological processes of CRC remain unknown. The present study used reverse transcription-quantitative polymerase chain reaction to determine the expression levels of microRNA (miR)-296 in CRC cell lines and tissues. In addition, a miR-296 mimic was transfected into CRC cells, and the effects of miR-296 on cell proliferation and apoptosis were explored by MTT assay and flow cytometry. Luciferase assays were also performed to validate arrestin $\beta 1$ (ARRB1) as a miR-296 target in SW480 and HCT-116 cells. The results demonstrated that miR-296 is a critical tumor suppressor which was downregulated in CRC patients. Increased expression levels of miR-296 were positively associated with a longer survival time of CRC patients. In addition, it was demonstrated that ARRB1 is a direct downstream target of miR-296. Upregulation of miR-296 in SW480 and HCT-116 cells resulted in suppressed cell growth and increased cell apoptosis through an ARRB1-dependent mechanism. Furthermore, the molecular mechanisms underlying the antitumor effect of miR-296 in CRC are at least in part due to the inactivation of the RAC- $\alpha$ serine/threonine-protein kinase (AKT) signaling pathway induced by the suppression of ARRB1 expression. Overall, the present study, to the best of the author's knowledge, is the first to demonstrate that the miR-296-ARRB1-AKT signaling pathway forms a critical feedback loop and mediates CRC carcinogenesis, and these findings may introduce a potential therapeutic strategy for the treatment of CRC.
\end{abstract}

\section{Introduction}

Colorectal cancer (CRC) represents the most commonly diagnosed tumor worldwide, and the mortality rate of CRC is

Correspondence to: Dr Chao Chen, Department of Gastrointestinal Surgery, Huizhou Municipal Central Hospital, 41 North Eling Road, Huizhou, Guangdong 516001, P.R. China

E-mail: chenchaopw@126.com

*Contributed equally

Key words: microRNA-296, colorectal cancer, apoptosis, arrestin $\beta 1$ higher in developed areas than in developing areas (1). Despite the current treatment strategies including surgery and radiochemotherapy resulting in an improved treatment outcome, the median survival time of patients with malignant CRC is only 20-24 months (2). Currently, mounting evidence indicates that $\mathrm{CRC}$ is an epidemiologically heterogeneous disease and the molecular mechanisms of CRC are not fully clarified (3). Therefore, identification of novel molecular biomarkers involved in mediating the pathogenesis of CRC and predicting the prognosis of CRC patients is of primary concern.

MicroRNAs (miRNA), a class of single-stranded noncoding RNAs, play a critical role in degrading target mRNAs through forming hybrids with the 3'-untranslated region sequences. Several studies have revealed that miRNAs serve as oncogenes or tumor suppressors in CRC carcinogenesis (4). miR-296, mapped to the human 20q13.32 gene locus, is characterized as a critical mediator which is involved in the biological processes of cell growth, angiogenesis and apoptosis $(5,6)$. Previously, miR-296 has been revealed to contribute to the development of various malignant tumors, such as ovarian, prostate, lung and breast cancers (7-10). It is noteworthy that Kunte et al (11) demonstrated that miR-296 is profoundly downregulated at the early stages of colon tumors and this may play a critical role in the pathological process of CRC neoplasia (11). However, its expression pattern, clinical significance, and biological functions in CRC remain unclear.

Arrestin $\beta 1$ (ARRB1), a mainstay of the $\beta$-arrestin family, has been revealed to serve as a multifunctional adaptor contributing to the mediation of several signaling pathways which are involved in cancer cellular progression (12-14). Emerging evidence demonstrates that ARRB1 may interact with histone acetyl-transferase, facilitating its recruitment to target histones, with consequent increased chromatin acetylation and transcription activation $(13,15,16)$. Although ARRB1 functions as a tumor oncogene that is associated with aggressive progression in various malignant cancers $(17,18)$, its biological significance in CRC has not been extensively elucidated.

The present study verified that miR-296 was significantly decreased in CRC patients. Upregulation of miR-296 in CRC cells resulted in decreased cell proliferation and increased cell apoptosis. Importantly, ARRB1 was confirmed as a novel target of miR-296, and it was elucidated that the miR-296ARRB1-RAC- $\alpha$ serine/threonine-protein kinase (AKT) axis contributes to regulation of tumor growth and cell apoptosis of SW480 and HCT-116 cells. 


\section{Materials and methods}

Tissue samples. CRC specimens were collected from 108 patients (60 male patients and 48 female patients; mean age, $53.32 \pm 11.32$ years; median age, 55 years) with CRC at Huizhou Municipal Central Hospital (Huizhou, China) from January 2008 to December 2014. CRC tissue use in the present study was approved by the Specialty Committee on Ethics of Biomedicine Research (PJ2008-012-03), Huizhou Municipal Central Hospital. In this study, human tissue acquisition and use complied with the National Regulations on the Use of Clinical Samples in China, and was performed in accordance with the ethical standards from the Declaration of Helsinki. All patients gave written informed consent. In the present study, subjects' rights and interests were fully protected. There was no potential risk to the subjects. The board of ethics committee agreed to the study work as planned. The follow-up was carried out in all patients, with survival time being censored in July 2014. The follow-up procedure occurred every 6 months by telephone and the final follow-up for patients occurred in January 2014. The selection criteria were as follows: i) The subject was diagnosed with CRC and had no history of other tumors; ii) complete clinical data was available for the subject, including age, sex, clinical manifestations and mean tumor diameter; iii) patients receiving chemotherapy or radiotherapy prior to surgery were excluded. The clinical characteristics of patients with CRC are presented in Table I.

Cell culture. Human CRC cell lines (HT-29, SW620, LoVo, SW480 and HCT-116) and the normal colon cell line NCM 460 were obtained from the American Type Culture Collection (Manassas, VA, USA) and cultured in Dulbecco's modified Eagle's medium (DMEM, Sigma-Aldrich; Merck KGaA, Darmstadt, Germany) supplemented with $10 \%$ fetal bovine serum (FBS; Gibco; Thermo Fisher Scientific, Inc., Waltham, MA, USA), $100 \mathrm{U} / \mathrm{ml}$ penicillin and $100 \mu \mathrm{g} / \mathrm{ml}$ streptomycin at $37^{\circ} \mathrm{C}$ in a humidified atmosphere containing $5 \% \mathrm{CO}_{2}$.

miRNAs and transfection. The miR-296 mimic, the miR-NC and the miR-296 inhibitor were purchased from Invitrogen (Thermo Fisher Scientific, Inc.). Sequences were as follows: miR-296 mimics, sense 5'-AGGGCCCCCCCUCAAUCC UGU-3', antisense 5'-ACAGGAUUGAGGGGGGGCCCU-3'; miR-NC, sense 5'-UUCUCCGAGGACGUCUGACUGUTT-3', antisense 5'-ACGUGACACGUUCGGAGGGTT-3'; miR-296 inhibitor, 5'-ACAGGCCGGACAAGUCGAAUG. The ARRB1 coding sequence was cloned into a pCDNA3.1 vector (Invitrogen; Thermo Fisher Scientific, Inc.), whereas a blank vector was used as a negative control. Short interfering RNA (siRNA) against ARRB1 (ARRB1 siRNA) (sense, 5'-CCGAC UCACAGUCCAUCAATT-3'; antisense, 5'-UUGAGGAUC UACGTACGGTT-3') and negative control with siRNA-negative control [green fluorescent protein (GFP)-siRNA] (sense, 5'-ACGCCAUUGGUAUCGUUTT-3'; antisense, 5'-AAGAC UAUTUCAAUGGUCCTT-3') were synthesized and purified by Shanghai GenePharma Co., Ltd. (Shanghai, China). Cells were transfected with the miRNA mimics, siRNA and pCDNA3.1-ARRB1 using Lipofectamine ${ }^{\circledR} 3000$ Reagent (Invitrogen; Thermo Fisher Scientific, Inc.), according to the manufacturer's protocol. For transfection, $2 \mu \mathrm{g}$ miR-296
Table I. Association between miR-296 expression and clinicopathological variables of colorectal cancer patients.

\begin{tabular}{|c|c|c|c|c|}
\hline \multirow[b]{2}{*}{ Characteristics } & \multirow[b]{2}{*}{ Value } & \multicolumn{2}{|c|}{$\begin{array}{c}\text { MicroRNA-296 } \\
\text { expression }\end{array}$} & \multirow[b]{2}{*}{ P-value } \\
\hline & & High & Low & \\
\hline No. of patients & 108 & 50 & 58 & \\
\hline Age, years & & & & 0.15 \\
\hline$<50$ & 64 & 30 & 34 & \\
\hline$\geq 50$ & 44 & 20 & 24 & \\
\hline Sex & & & & 0.14 \\
\hline Male & 60 & 28 & 32 & \\
\hline Female & 48 & 22 & 26 & \\
\hline MTD, cm & & & & 0.18 \\
\hline$<5 \mathrm{~cm}$ & 77 & 35 & 42 & \\
\hline$\geq 5 \mathrm{~cm}$ & 31 & 15 & 16 & \\
\hline Tumor location & & & & 0.11 \\
\hline Rectum & 46 & 20 & 26 & \\
\hline Colon & 62 & 30 & 32 & \\
\hline Distant metastasis & & & & 0.02 \\
\hline Present & 41 & 24 & 17 & \\
\hline Absent & 67 & 26 & 41 & \\
\hline Lymph node metastasis & & & & $<0.01$ \\
\hline Present & 31 & 6 & 25 & \\
\hline Absent & 77 & 54 & 23 & \\
\hline TNM stage & & & & $<0.01$ \\
\hline II & 43 & 31 & 12 & \\
\hline III/IV & 65 & 19 & 46 & \\
\hline
\end{tabular}

MTD, mean tumor diameter; TNM, T category describes the primary tumor site; $\mathrm{N}$ category describes the regional lymph node involvement; $M$ category describes the presence or otherwise of distant metastatic spread. Bold indicates significant differences.

mimic, miR-NC, miR-296 inhibitor, pcDNA3.1, pcDNA3.1ARRB1, ARRB1 siRNA or GFP-siRNA vectors were diluted in $200 \mu 1$ Opti-MEM (Thermo Fisher Scientific, Inc.) and were incubated at room temperature for $5 \mathrm{~min}$. Subsequently, the diluted vectors and Lipofectamine 3000 were combined and incubated for a further $20 \mathrm{~min}$ at room temperature, prior to their addition to cells $\left(4 \times 10^{5} /\right.$ well) seeded in 6 -well plates. After transfection for $6 \mathrm{~h}$, the culture medium was replaced with RPMI-1640 medium containing 10\% FBS (HyClone; GE Healthcare Life Sciences, Logan, UT, USA), followed by incubation for a further $24 \mathrm{~h}$ at $37^{\circ} \mathrm{C}$ and $5 \% \mathrm{CO}_{2}$. At $48 \mathrm{~h}$ post-transfection, the cells were used in subsequent experiments. ARRB1 was predicted as a direct downstream target of miR-296 by Target Scan 7.2 database (http://www.targetscan.org).

Cell proliferation assay. 3-(4, 5-dimethylthiazol-2-yl)-2, 5-diphenyltetrazolium bromide (MTT) assay was used to assess cell viability. Cells were seeded in 96-well plates and were incubated with $0.2 \mathrm{mg} / \mathrm{ml} \mathrm{MTT}$ for $4 \mathrm{~h}$ at $37^{\circ} \mathrm{C}$. After removal of the medium, the formazan crystals produced by 
live cells were dissolved in $150 \mu \mathrm{l}$ dimethyl sulfoxide (SigmaAldrich; Merck KGaA) and the absorbance of each sample was read at a wavelength of $570 \mathrm{~nm}$ using an Ultra multi-functional microplate reader (Tecan US, Inc., Morrisville, NC, USA).

Reverse transcription-quantitative polymerase chain reaction (RT-qPCR) analysis. Tumor specimens were frozen at $-75^{\circ} \mathrm{C}$ to use. Total RNA, including miRNA, was extracted from cells/ tissues with TRIzol ${ }^{\circledR}$ reagent (Invitrogen; Thermo Fisher Scientific, Inc.), and RNA was reverse transcribed into cDNA using a qPCR RT kit (FSQ-101; Toyobo Life Science, Osaka, Japan), according to the manufacturer's protocol. cDNA was amplified by Platinum SYBR Green qPCR SuperMix-UDG (Invitrogen; Thermo Fisher Scientific, Inc.). RT-qPCR was performed according to the manufacturer's protocol. The PCR conditions were $10 \mathrm{~min}$ at $95^{\circ} \mathrm{C}, 1 \mathrm{~min}$ at $55^{\circ} \mathrm{C}, 40$ cycles of $15 \mathrm{sec}$ at $95^{\circ} \mathrm{C}$, followed by $30 \mathrm{sec}$ at $55^{\circ} \mathrm{C}$. For mRNA analysis, RT-qPCR was performed using the Platinum SYBR Green qPCR SuperMix-UDG (Qiagen GmbH, Hilden, Germany) using the 7500 Fast Real-time PCR system (Applied Biosystems; Thermo Fisher Scientific, Inc.). The relative fold expression of the target gene was normalized to $\beta$-actin and was calculated according to the $2^{-\Delta \Delta C q}$ method (19). The PCR primer sequences for ARRB1 (forward, 5'-CCTGGAT GTCTTGGGTCTG-3' and reverse, 5'-TGATGGGTTC TCCGTGGTA-3') were as previously described (20). The primer sequences for $\beta$-actin were as follows: Forward, 5'-ACCAACTGGGACGACATGGAGAAA-3' and reverse, 5'-TAGCACAGCCTGGATAGCAACGTA-3'.

Northern blot analysis. Northern blot analysis was performed as described previously (21). Antisense RNA probes were 3'-end-labeled with 32P- $\gamma$ ATP by T4 polynucleotide kinase; miR-296 antisense probe sequence 5'-CCCUUCCAGAGGGCCCCCCCUCAAU CCUGUUGUGCCUA-3' and U6 spliceosomal RNA antisense probe sequence 5'-GCAGGGGCCATGCTAATCT TCTCTGTATCG-3' were used.

Luciferase reporter assay. Cells were seeded in 24-well plates and incubated for $24 \mathrm{~h}$ prior to transfection. The ARRB1-3'untranslated region (UTR)-WT (wild-type) or ARRB1-3'UTR-MT (mutant) were transfected with a miR-296 mimic or miR-NC into cells. For the luciferase reporter assay, $3 \times 10^{4}$ cells were seeded into 48 -well plates overnight, followed by transfection with miR-296 (50 pmol/ml), Renilla luciferase pRL-TK vector $(100 \mathrm{ng} / \mathrm{ml}$; Promega Corporation, Madison, WI, USA) and pGL3 plasmid with WT/MT 3'UTR of ARRB1 gene using Lipofectamine ${ }^{\circledR}$ 2000. After transfection for $24 \mathrm{~h}$, the luciferase activity was determined by the Dual Luciferase Reporter Assay System (Promega Corporation). Renilla luciferase activities were normalized to firefly luciferase activities.

Apoptosis assay. Cell apoptosis was assessed with ApoScreen Annexin V Apoptosis kit (BenderMedSystems, GmbH, Vienna, Austria). After being washed twice with PBS, cell samples were collected and fluorescein isothiocyanate added. All cells were analyzed by flow cytometry (FACScan; BD Biosciences, Franklin Lakes, NJ, USA). Analyses were performed using
CellQuest software version 5.0 (BD Biosciences). Experiments were conducted triple times.

Protein extraction and western blotting. Western blot analysis was conducted according to protocols described in a previous study (22). The protein concentration of cell lysates was quantified using a bicinchoninic acid kit (Beyotime Institute of Biotechnology, Haimen, China), and $50 \mu \mathrm{g}$ protein for each sample was separated by SDS-PAGE on $10 \%$ gels and was then transferred to a polyvinylidene fluoride (PVDF) membrane (EMD Millipore, Billerica, MA, USA). The membranes were blocked in 5\% non-fat milk diluted with Tris-buffered saline-Tween (TBST) (10 mM Tris-HCl, pH 7.5; $150 \mathrm{mM} \mathrm{NaCl} ; 0.1 \%$ Tween-20) at room temperature for $1 \mathrm{~h}$ and were incubated overnight at $4^{\circ} \mathrm{C}$ with primary antibodies. Primary antibodies used were against ARRB1 (catalog no. sc-377015; 1:500; Santa Cruz Biotechnology, Inc., Dallas, TX, USA), AKT (catalog no. GTX110613; 1:600; GeneTex, Inc., Irvine, CA, USA), B cell lymphoma (Bcl)-2 (catalog no. sc-20067; 1:500; Santa Cruz Biotechnology, Inc.), signal transducer and activator of transcription 3 (STAT3) (catalog no. ab-11935; 1:500; Abcam, Cambridge, MA, USA), Bcl-2associated X protein (Bax) (catalog no. sc-23959; 1:500; Santa Cruz Biotechnology, Inc.), Bcl-2 homologous antagonist/killer (Bak) (catalog no. sc-7873; 1:500; Santa Cruz Biotechnology, Inc.), NADPH oxidase activator (Noxa) (catalog no. 14766; 1:1,000; Cell Signaling Technology Inc., Danvers, MA, USA), p53 upregulated modulator of apoptosis (PUMA) (catalog no. 24633; 1:1,000; Cell Signaling Technology, Inc.) and $\beta$-actin (catalog no. ab8227; 1:1,000; Abcam). The membranes were incubated with horseradish peroxidase-conjugated goat anti-rabbit (catalog no. ab6721; 1:1,000; Abcam) or rabbit anti-mouse immunoglobulin $\mathrm{G}$ secondary antibodies (catalog no. ab6728; 1:1,000; Abcam) for $2 \mathrm{~h}$ at room temperature. The proteins were visualized using Enhanced Chemiluminescence Plus reagents (Amersham; GE Healthcare, Chicago, IL, USA), and band density was measured using ImageJ software (National Institutes of Health, Bethesda, MD, USA), and the values were normalized to the densitometric values of $\beta$-actin in each sample.

Transmission electron microscopy (TEM). Cultured cells were fixed for $2 \mathrm{~h}$ at $4^{\circ} \mathrm{C}$ with $2.5 \%$ glutaraldehyde in $0.2 \mathrm{M}$ caco-

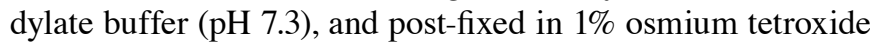
in $0.45 \mathrm{M}$ cacodylate buffer ( $\mathrm{pH}$ 7.3). The fixed material was dehydrated in a graded series of alcohol and embedded in Epon 812. For light microscopy, semi-thin sections were stained with Toluidine Blue and observed under a Reichert microscope equipped with Nomarski optics. Images were captured using a 12-bit charge-coupled device camera. For TEM, ultra-thin sections were classically contrasted with Ultracut $\mathrm{S}$ and were stained with uranyl acetate and lead citrate for 30 and 5 min at room temperature, respectively. The stained sections were observed under a JEOL transmission electron microscope (JEOL, Ltd., Tokyo, Japan) at x1200 magnification.

Immunohistochemistry. Human tissues were fixed in $4 \%$ formalin for $4 \mathrm{~h}$ at room temperature, and were then embedded in paraffin and cut into $3-\mu \mathrm{m}$ sections. The sections were then deparaffinized in xylol for $20 \mathrm{~min}$ at $60^{\circ} \mathrm{C}$ and rehydrated in a 
graded ethanol series $(75 \%$ alcohol for $1.5 \mathrm{~h}, 95 \%$ alcohol for $1.5 \mathrm{~h}, 95 \%$ alcohol for $1 \mathrm{~h}$, anhydrous ethanol for $2.5 \mathrm{~h}$ ). Antigen retrieval was performed by heating the samples in a microwave for $20 \mathrm{~min}$ in $1 \mathrm{mM}$ EDTA buffer ( $\mathrm{pH} \mathrm{8.0)}$. Thereafter, endogenous peroxidase activity was quenched by $30 \mathrm{~min}$ incubation in $3 \%$ methanolic hydrogen peroxide solution at room temperature. The sections were incubated in nonimmune serum (Invitrogen; Thermo Fisher Scientific, Inc.) for $30 \mathrm{~min}$ and were then incubated overnight at $4^{\circ} \mathrm{C}$ in ARRB1 primary monoclonal antibody (cat. no., ab31868; 1:500; Abcam). After washing in TBST, the immunolabeled sections were incubated with a horseradish peroxidase-conjugated secondary antibody (catalog no. AS09 602; 1:500; Agrisera AB, Vännäs, Sweden) for $20 \mathrm{~min}$ at room temperature, and then with peroxidaseconjugated complex (Dako, Glostrup, Denmark) for $20 \mathrm{~min}$. Finally, the sections were visualized with 3,3'-diaminobenzidine and counterstained with hematoxylin. To ensure the specificity of the immunostaining, negative controls were prepared by replacing the primary antibody with non-immune serum. Two independent pathologists examined five random fields (1 field $=0.159 \mathrm{~mm}^{2}$ at $\times 100$ magnification; Leica DMRX; Leica Microsystems, Inc., Buffalo Grove, IL, USA) in each sample and scored each sample without knowledge of patient outcome (double-blinded).

Statistical analysis. All statistical analyses were performed using GraphPad Prism 5.0 software (GraphPad Software, Inc., La Jolla, CA, USA). Experimental data are presented as the mean \pm standard deviation from triplicate experiments. A Student's t-test was used to analyze differences between two groups. A one-way analysis of variance and Tukey's post hoc test was performed to detect statistical differences among multiple groups. Pearson's $\chi^{2}$ tests were used for analysis of the association between clinicopathological features and miR-296 expression in CRC patients. The correlation between miR-296 and ARRB1 expression was examined by Spearman's correlation coefficient. Overall survival (OS) curves were calculated using the Kaplan-Meier method and were compared using the log-rank test. Possible prognostic factors were analyzed by univariate analysis for their potential association with OS. Factors with a $\mathrm{P}<0.05$ in the univariate analysis were included in the multivariate analysis, in order to analyze their potential association with OS using Cox regression with the backward stepwise method. $\mathrm{P}<0.05$ was considered to indicate a statistically significant difference.

\section{Results}

miR-296 is frequently downregulated in CRC specimens. The present study first used RT-qPCR to measure the miR-296 expression in $28 \mathrm{CRC}$ tissues and corresponding adjacent normal specimens. It was demonstrated that miR-296 downregulation was detected in 25/28 (89.28\%) CRC tumors (Fig. 1A), which indicated that the decrease of miR-296 is a frequent event in CRC. The RT-qPCR data revealed that miR-296 expression in the tumors was approximately 2.8 -fold lower compared with adjacent normal tissues (4.98 \pm 1.02 vs. $1.99 \pm 0.54 ; \mathrm{P}<0.05$; Fig. 1B). The mean miR-296 expression level of CRC tissues was 4.98, which was utilized to divide CRC patients into either a low expression group $(<4.98, n=58)$
Table II. Univariate analysis of factors associated with survival of colorectal cancer patients.

\begin{tabular}{lccc}
\hline & \multicolumn{3}{c}{ Survival } \\
\cline { 2 - 4 } Variable & HR & $95 \%$ CI & P-value \\
\hline Sex (male vs. female) & 0.758 & $0.397-1.076$ & 0.417 \\
Age $(\geq 50$ vs.<50) & 0.812 & $0.276-1.374$ & 0.398 \\
MTD ( $\geq 5$ cm vs. $<5 \mathrm{~cm})$ & 0.541 & $0.322-1.179$ & 0.743 \\
MicroRNA-296 (low vs. high) & 1.843 & $1.031-2.458$ & $0.012^{\mathrm{a}}$ \\
Tumor location & 0.742 & $0.512-1.592$ & 0.732 \\
TNM stage & 0.965 & $0.651-1.868$ & $0.032^{\mathrm{a}}$ \\
Lymph node metastasis & 0.713 & $0.251-0.964$ & $0.021^{\mathrm{a}}$ \\
Distant metastasis & 0.433 & $0.113-0.879$ & $0.006^{\mathrm{a}}$
\end{tabular}

Univariate analysis, Cox proportional hazards regression model. ${ }^{a} \mathrm{P}<0.05$ was considered to indicate a statistically significant difference. MTD, mean tumor diameter; TNM, T category describes the primary tumor site; $\mathrm{N}$ category describes the regional lymph node involvement; $\mathrm{M}$ category describes the presence or otherwise of distant metastatic spread; HR, hazard ratio; CI, confidence interval.

or a high expression group $(\geq 4.98, n=50)$. The present study then investigated the association between miR-296 expression and clinicopathological parameters (Table I). The expression level of miR-296 was inversely associated with tumor node metastasis $(\mathrm{TNM})$ stage $(\mathrm{P}<0.01)$, lymph node metastasis $(\mathrm{P}<0.01)$ and distant metastasis $(\mathrm{P}=0.02)$. No significant association was identified between miR-296 expression and other clinical parameters.

Univariate survival analysis revealed a significant association between expression of miR-296, TNM stage $(\mathrm{P}=0.032)$, distant metastasis $(\mathrm{P}=0.006)$, lymph node metastasis $(\mathrm{P}=0.021)$ and recurrence-free survival rate (Table II), while no significant association was observed between recurrence-free survival rate and sex, age, tumor size, or tumor location. Multivariate analysis using the Cox proportional hazards model for all variables included in the univariate analysis further revealed that expression of miR-296 $(\mathrm{P}=0.008)$, TNM stage $(\mathrm{P}=0.021)$, distant metastasis $(\mathrm{P}=0.007)$, and lymph node metastasis $(\mathrm{P}=0.002)$ at diagnosis were independent prognostic factors for patients with CRC (Table III).

Next, the present study investigated the prognostic value of miR-296 between high and low expression groups using the Kaplan-Meier survival method. These results demonstrated that the patients with high expression levels of miR-296 exhibited a significantly prolonged overall survival (OS) compared with those with low expression levels of miR-296 $(\mathrm{P}<0.05$; Fig. 1C).

The present study further measured the miR-296 expression in one normal colon cell line NCM 460 and five CRC cell lines, including HT-29, SW620, LoVo, SW480 and HCT-116 by RT-qPCR analysis (Fig. 1D) and Northern blotting (Fig. 1E). SW480 and HCT-116 cells revealed a significantly lower expression level of miR-296 compared with HT-29, SW620, LoVo and NCM 460 cells. Therefore, SW480 and HCT-116 cells were selected for use in subsequent experiments. 
A

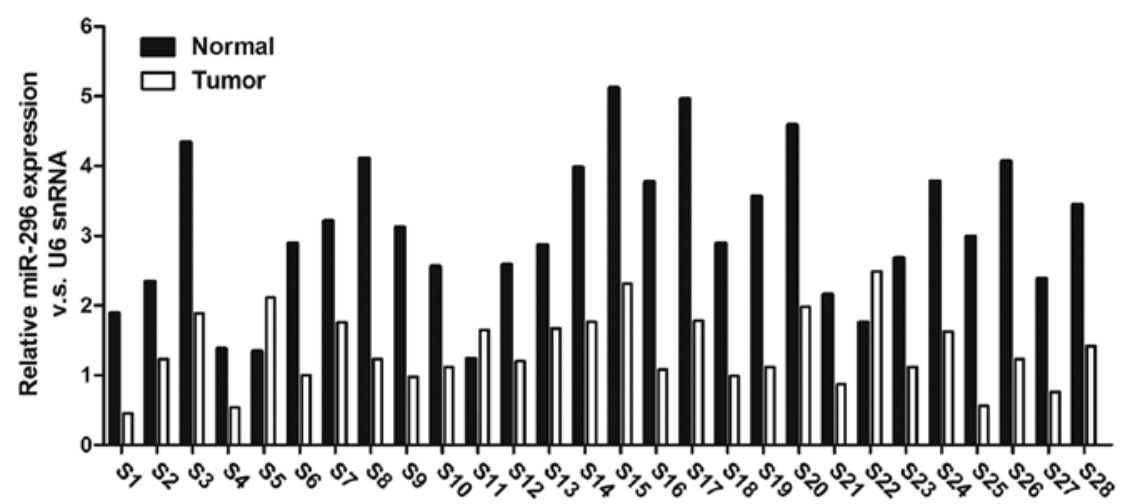

B
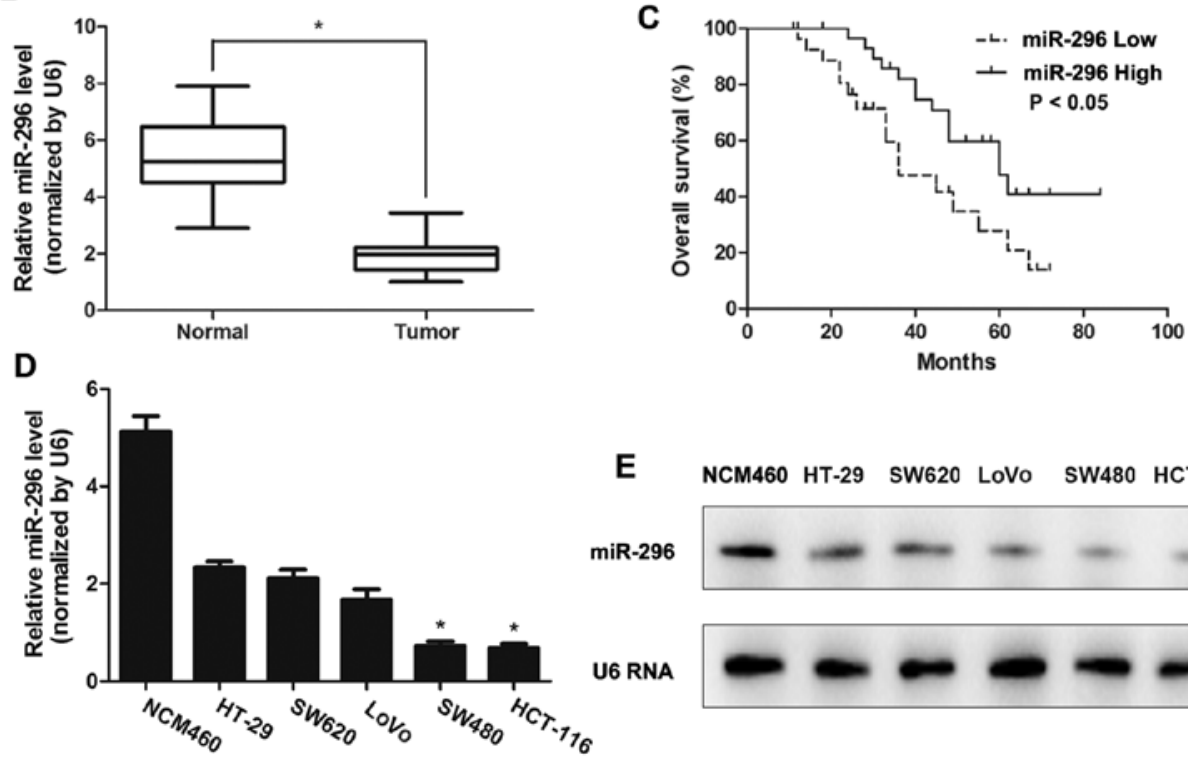

NCM460 HT-29 SW620 LoVo SW480 HCT-116

Figure 1. Expression levels of miR-296 in CRC tissues and cell lines. (A) miR-296 expression in 28 CRC tissues and corresponding adjacent normal tissues as determined by RT-qPCR analysis. (B) miR-296 expression was measured by RT-qPCR in adjacent normal tissues and CRC tissues. All expression levels were normalized to the level of U6 snRNA expression. "P<0.05. (C) Kaplan-Meier survival curves according to miR-296 expression (P<0.001). (D) RT-qPCR analysis indicated that the expression level of miR-296 was significantly lower in SW480 and HCT-116 cells compared to the normal colon cell line NCM 460. " $\mathrm{P}<0.05$ vs. the normal colon cell line NCM 460. (E) NB analysis revealed similar results; NB analyses were performed in triplicates, and representative results are shown. miR, microRNA; CRC, colorectal cancer; NB, Northern blot; RT-qPCR, reverse transcription-quantitative polymerase chain reaction; sn, small nuclear.

Overexpression of miR-296 inhibits cell survival and promotes cell apoptosis of CRC cells in vitro. To investigate the antitumor effects of miR-296 in CRC cells, the present study upregulated the miR-296 expression level by treating with miR-296 mimics. As presented in Fig. 2A, transfection of SW480 and HCT-116 cells with miR-296 mimics resulted in a significant increase in miR-296 expression. Consistent with the previously demonstrated critical role of miR-296 in cell survival, miR-296 mimics-transfected SW480 and HCT-116 cells (overexpressing miR-296) revealed significantly lower survival rates compared with miR-NC transfected cells as evidenced by MTT assay (Fig. 2B). In addition, upregulation of miR-296 expression in SW480 and HCT-116 cell lines enhanced CRC cell apoptosis as measured by flow cytometry assays (Fig. 2C). Furthermore, TEM analysis revealed classical apoptotic changes in SW480 and HCT-116 cells transfected with miR-296 mimics, such as nuclear condensation and fragmentation and cell blebbing morphological alterations (Fig. 2D). These data strongly suggested that miR-296 suppressed CRC cell survival through enhancing cell apoptosis.
Table III. Multivariate analyses of factors associated with survival of colorectal patients.

\begin{tabular}{lccc}
\hline Variable & HR & $95 \%$ CI & P-value \\
\hline MicroRNA-296 (low vs. high) & 1.765 & $1.239-3.581$ & $0.008^{\mathrm{a}}$ \\
TNM stage & 1.132 & $0.542-1.783$ & $0.021^{\mathrm{a}}$ \\
Lymph node metastasis & 2.349 & $0.396-3.486$ & $0.002^{\mathrm{a}}$ \\
Distant metastasis & 2.132 & $0.987-3.783$ & $0.007^{\mathrm{a}}$ \\
\hline
\end{tabular}

Multivariate analysis, Cox proportional hazards regression model; backward stepwise method. ${ }^{a} \mathrm{P}<0.05$ was considered to indicate a statistically significant difference. TNM, T category describes the primary tumor site; $\mathrm{N}$ category describes the regional lymph node involvement; $\mathrm{M}$ category describes the presence or otherwise of distant metastatic spread HR, hazard ratio; CI, confidence interval.

miR-296-induced apoptosis is associated with dephosphorylation of AKT. The AKT signaling pathway plays an important 


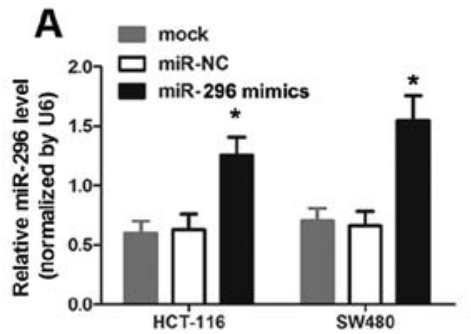

C
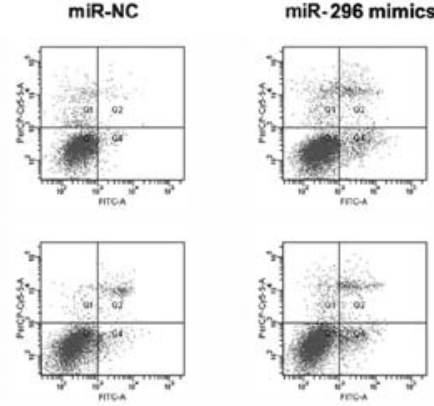

$\square$ miR-NC

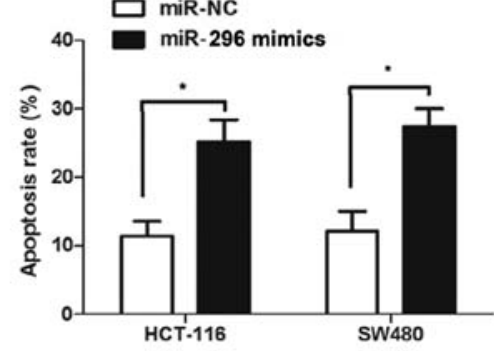

miR-296 mimics

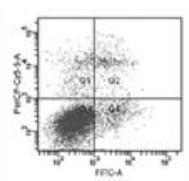

SW480

D

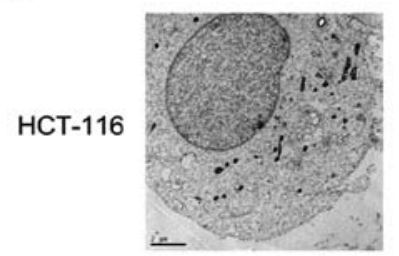

B

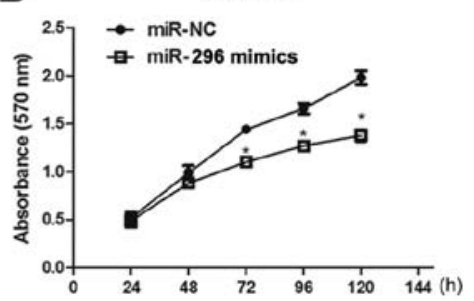

E

HCT-116

miR-NC miR-296 mimics

p-AKT

AKT

p-STAT3

STAT3

p-mTOR

mTOR

$\mathrm{Bcl}-2$

Bak

Noxa

Bax

Puma

$\beta$-actin

miR-296 mimics

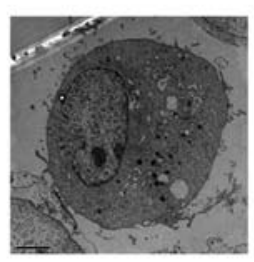

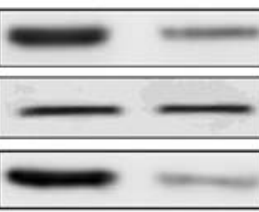
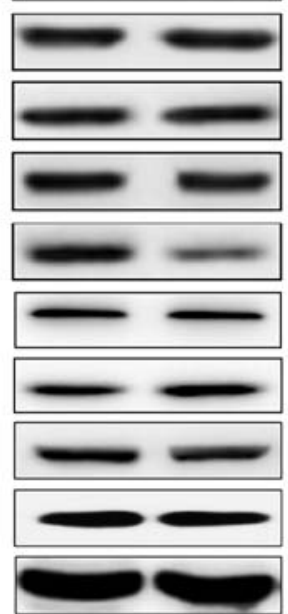

miR-NC

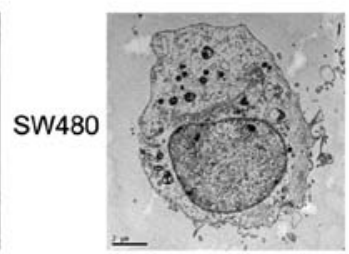

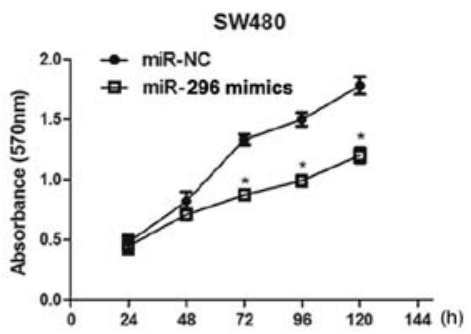

SW480

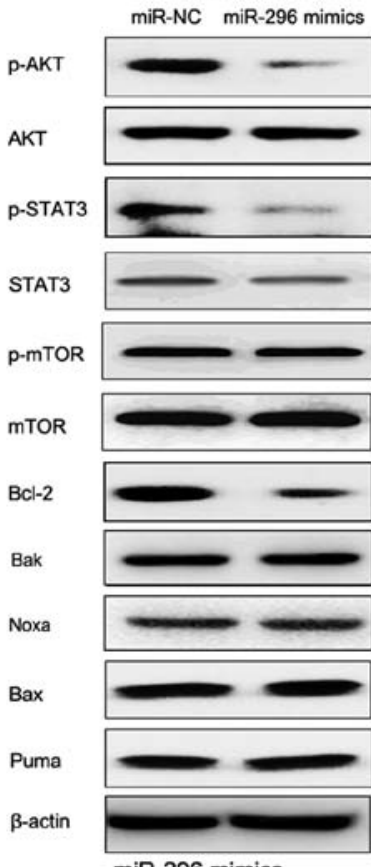

miR-296 mimics

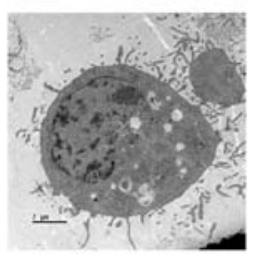

Figure 2. Effects of miR-296 on cell proliferation, cell apoptosis and the expression of associated molecules in SW480 and HCT-116 cell lines. (A) Reverse transcription-quantitative polymerase chain reaction analysis demonstrated that miR-296 expression was significantly enhanced in SW480 and HCT-116 cells transfected with miR-296 mimics compared to cells transfected with miR-NC. * $\mathrm{P}<0.05$ vs. miR-NC-transfected cells. (B) Effect of miR-296 upregulation on SW480 and HCT-116 cell proliferation as measured by MTT assay. ${ }^{*} \mathrm{P}<0.05$ vs. miR-NC-transfected cells. (C) Effect of miR-296 upregulation on SW480 and HCT-116 cell apoptosis rates. The number of apoptotic cells was significantly higher in SW480 and HCT-116 cells overexpressing miR-296 as measured by propidium iodide staining and flow cytometry. ${ }^{*} \mathrm{P}<0.05$. (D) Morphological analysis of SW480 and HCT-116 cell apoptosis induced by miR-296 mimics. The morphology of colorectal cancer cells were imaged by TEM. Magnification in transmission electron microscopy analysis was x6,000. (E) Altered AKT signaling and apoptosis are associated with overexpression of miR-296. Detection of p-AKT, AKT, p-STAT3, STAT3 and apoptosis-associated proteins in SW480 and HCT-116 cells was performed by western blotting. miR, microRNA; NC, negative control; p, phosphorylated; AKT, RAC- $\alpha$ serine/threonineprotein kinase; STAT3, signal transducer and activator of transcription 3; mTOR, serine/threonine-protein kinase mTOR; Bcl-2, B cell lymphoma 2; Bak, Bcl-2 homologous antagonist/killer; Bax, Bcl-2 associated X, apoptosis regulator; Noxa, NADPH oxidase activator; Puma, p53 upregulated modulator of apoptosis.

role in controlling CRC cellular processes, such as apoptosis and proliferation. Signal transducer and activator of transcription 3 (STAT3) has been reported to mediate cell survival and apoptosis in CRC cells through the AKT signaling pathway. To determine whether AKT pathway activation is involved in miR-296-induced apoptosis, the expression and activities of AKT signaling pathway molecules were examined in cellular apoptosis pathways. Western blot analysis revealed that AKT phosphorylation and STAT3 phosphorylation were significantly lower in SW480 and HCT-116 cells expressing the miR-296 mimics compared with cells transfected with
miR-NC, while no changes in the expression levels of total AKT, total STAT3, p-serine/threonine-protein kinase mTOR (mTOR) and total mTOR were observed (Fig. 2E). Moreover, a significant decrease in the protein expression level of anti-apoptotic protein Bcl-2 in SW480 and HCT-116 cells overexpressing miR-296 was observed, whereas the protein levels of the apoptotic proteins Bax, Bak, Noxa and PUMA did not appear to differ between the two groups (Fig. 2E). These results suggested that AKT, STAT3 and Bcl-2 are critical downstream effectors of miR296-induced apoptosis and suppression of proliferation in SW480 and HCT-116 cells. 
ARRB1 3' UTR (WT) 5' ...UCCAGUUUCUACCAGGGGGCCCC. hsa-miR-296 || || ||| CUCCCCCCCGGGA... ARRB1 3' UTR (MT) 5' ...UCCAGUUUCUACCA $\underline{G} \underline{G} \underline{G} \underline{C} C C C C .$.

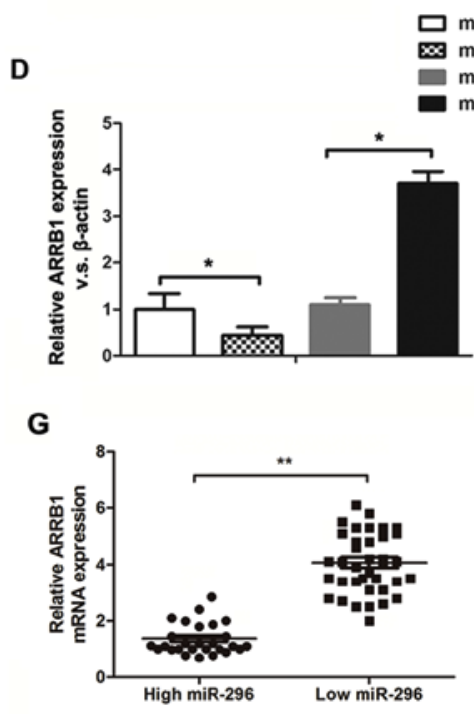

B

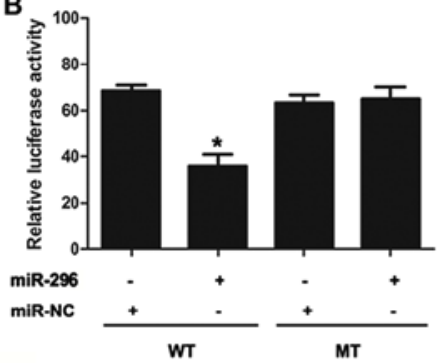

E

miR-NC
C
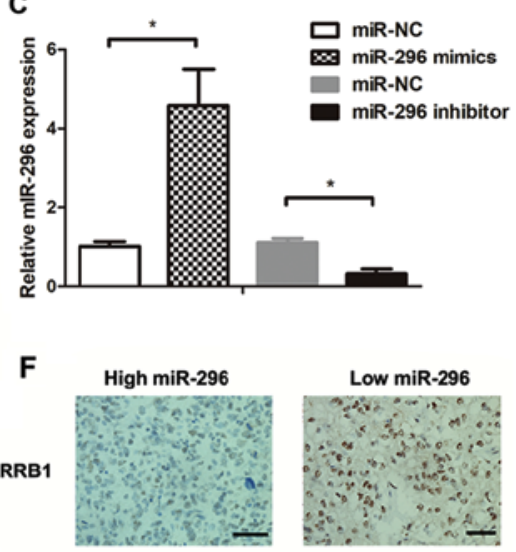

Low miR-296

ARRB1

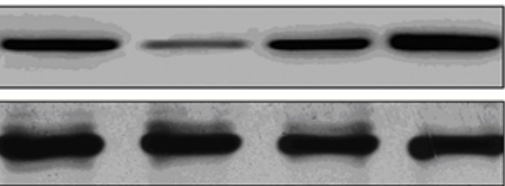

$\beta$-actin

H
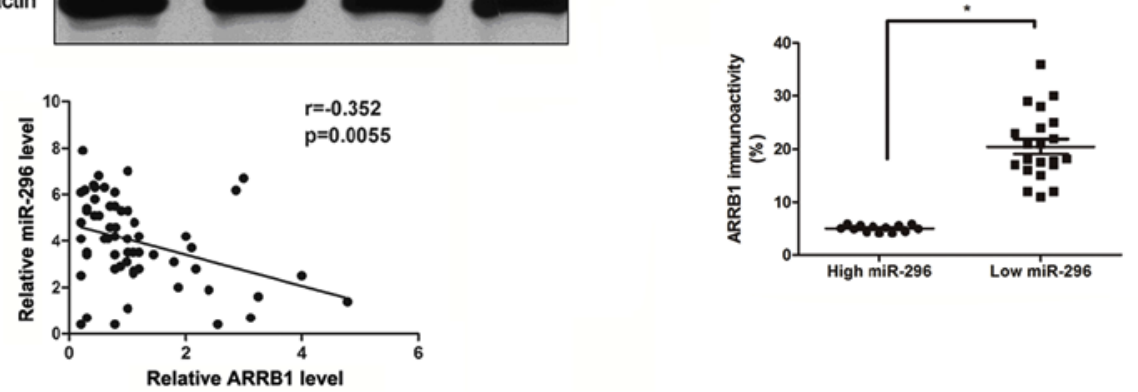

Figure 3. ARRB1 is a novel target of miR-296. (A) The putative miR-296 binding sequences in ARRB1 3'-UTR. (B) A significant decrease in the relative luciferase activity was observed following co-transfection of ARRB1-3'-UTR with the miR-296 mimics * P<0.05 vs. miR-NC-transfected cells. (C) RT-qPCR analysis of miR-296 expression levels after the introduction of mimic control, miR-296 mimic, inhibitor control, or miR-296 inhibitor to sw480 cells. ${ }^{*} \mathrm{P}<0.05$. (D) RT-qPCR and (E) western blotting analysis of ARRB1 protein levels following the introduction of mimic control, miR-296 mimic, inhibitor control, or miR-296 inhibitor to SW480 cells. * $\mathrm{P}<0.05$. (F) Representative immunostaining showed negative expression of ARRB1 in miR-296 high-expressing CRC tissue and positive expression of ARRB1 in miR-296 low-expressing CRC tissue. The expression of ARRB1 protein in miR-296 high-expressing tumors was significantly lower than that in miR-296 low-expressing tumors. Scale bar, $10 \mu \mathrm{m}$. ${ }^{*} \mathrm{P}<0.05$. (G) The expression of ARRB1 mRNA in miR-296 high-expressing tumors was significantly lower than that in miR-296 low-expressing tumors. ${ }^{*} \mathrm{P}<0.01$. (H) A significant inverse correlation between the mRNA levels of miR-296 and ARRB1 was observed in CRC tissues. P=0.0055. miR, microRNA; NC, negative control; UTR, untranslated region; CRC, colorectal cancer; WT, wild-type; MT, mutated; ARRB1, arrestin $\beta 1$.

Upregulation of miR-296 expression in CRC cells may inhibit the activity of the AKT-STAT3 signaling pathway, which ultimately results in promoting CRC cell apoptosis by suppressing levels of Bcl-2, an important anti-apoptotic protein.

ARRB1 is a direct target of miR-296. Since ARRB1 was predicted as a direct downstream target of miR-296 using the TargetScan 7.2 database (http://www.targetscan.org) (Fig. 3A), the present study next validated whether miR-296 regulates ARRB1 expression by using a luciferase reporter gene assay. As presented in Fig. 3B, overexpression of miR-296 in SW480 cells significantly suppressed the luciferase activity of the WT 3'-UTR of ARRB1, whereas no change in the luciferase activity of the Mut 3'-UTR of ARRB1was observed. RT-qPCR revealed that miR-296 mimics resulted in a significant increase in miR-296 expression and miR-296 inhibitor caused a significant decrease in miR-296 expression in SW480 cells (Fig. 3C). In addition, upregulation of miR-296 expression substantially decreased endogenous ARRB1 mRNA and protein levels in SW480 cells expressing miR-296 mimics compared with cells expressing only miR-NC, detected using RT-qPCR and western blot analysis (Fig. 3D and E). In contrast to SW480 cells overexpressing miR-296, SW480 cells underexpressing miR-296 showed enhanced ARRB1 mRNA and protein expression levels compared with the control (Fig. 3D and E). Furthermore, the association between miR-296 level and ARRB1 expression in CRC tissues was investigated, to confirm that ARRB1 was a novel target of miR-296. Immunohistochemical (ICH) staining indicated that ARRB1 expression in CRC with high expression level of miR-296 was significantly lower than in CRC with low miR-296 expression ( $\mathrm{P}<0.05$; Fig. 3F). RT-qPCR analysis revealed that ARRB1 mRNA expression was lower in the miR-296 high-expressing tumors compared with miR-296 low-expressing tumors, consistent with results from $\mathrm{ICH}$ ( $\mathrm{P}<0.05$; Fig. 3G). As depicted in Fig. 3H, an inverse correlation was observed between ARRB1 and miR-296 expression levels in human $\mathrm{CRC}$ tissues $(\mathrm{r}=-0.352, \mathrm{P}=0.0055)$. Therefore, the aforementioned results suggested that miR-296 reduced ARRB1 expression by targeting its 3'-UTR and ARRB1 is a direct downstream target of miR-296 in CRC.

ARRBl functions as an oncogene in CRC. The present study examined ARRB1 mRNA and protein expression levels in HT-29, SW620, LoVo, SW480 and HCT-116 cell lines by RT-qPCR and western blot analysis (Fig. 4A and B). As predicted, SW480 and HCT-116 cells exhibited significantly increased ARRB1 expression levels compared with HT-29, SW620, and LoVo cells, at both the mRNA and protein level. Therefore, SW480 and HCT-116 cells were selected for subsequent in vitro experiments. Western blot analysis revealed 
A

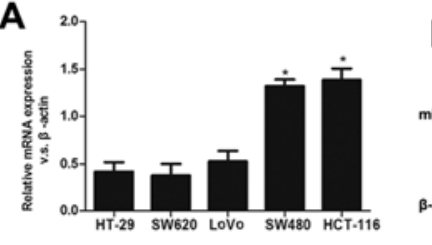

C
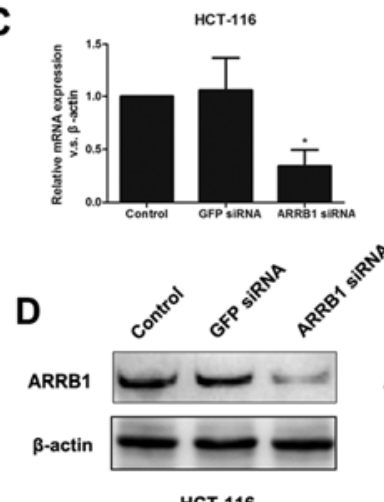

HCT-116

E
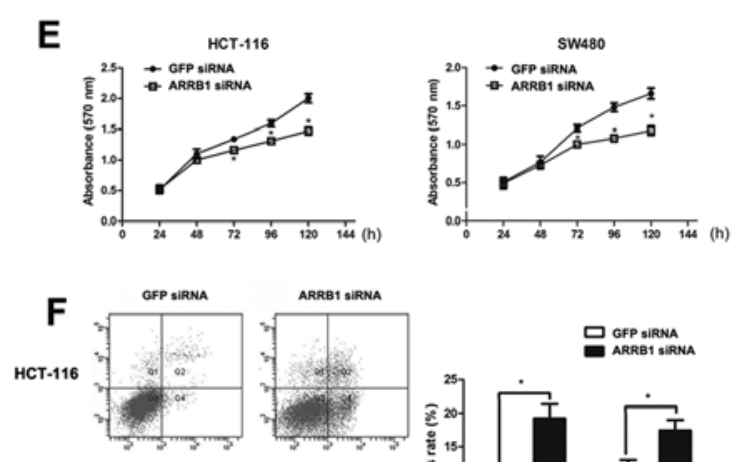

sw480
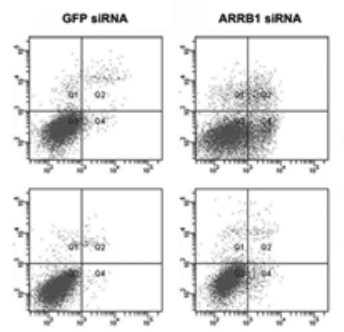

B

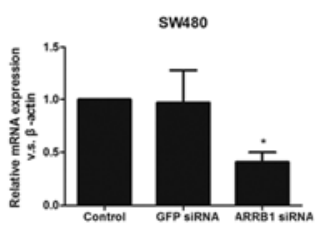

SW480
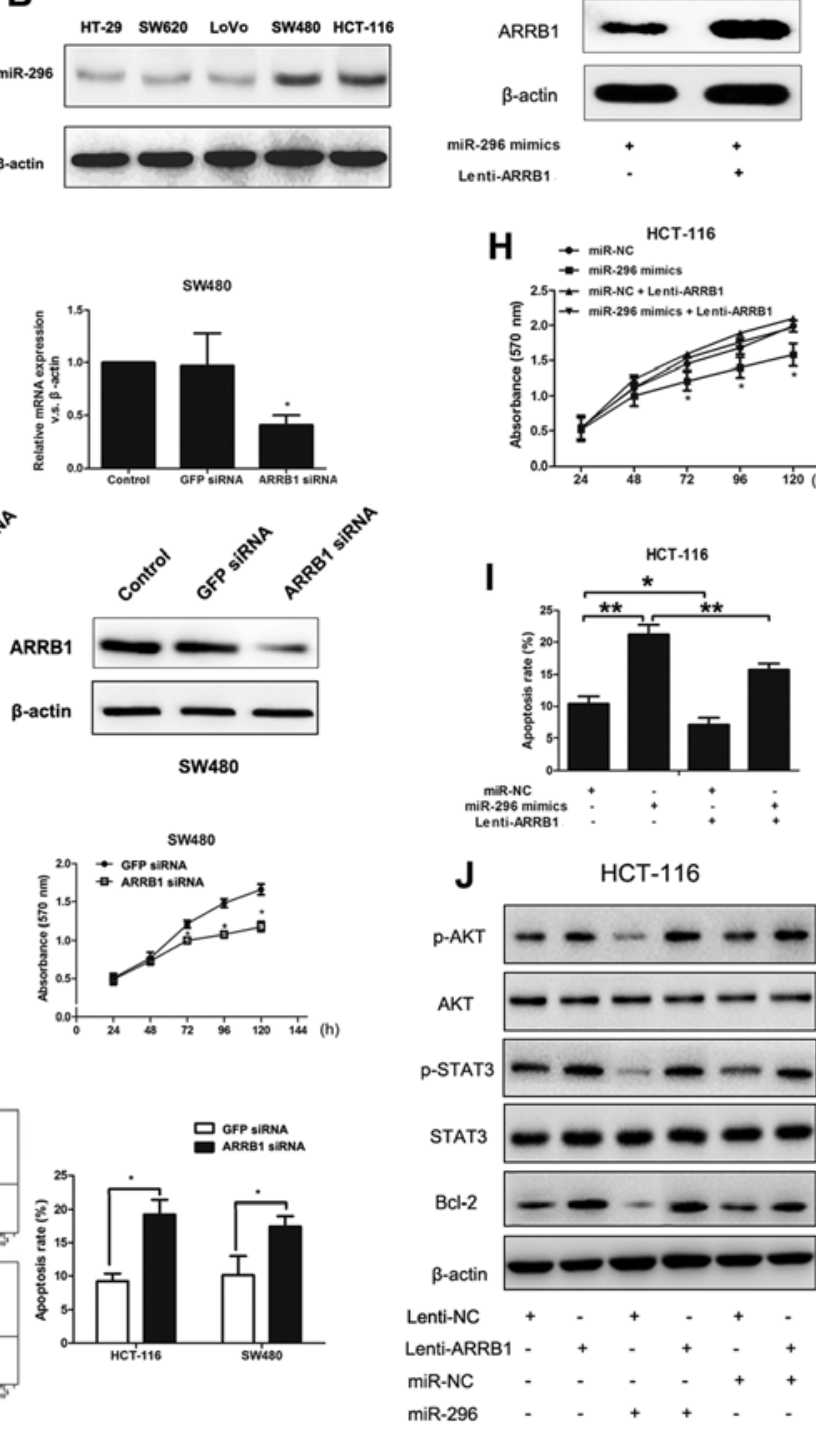

\section{I}

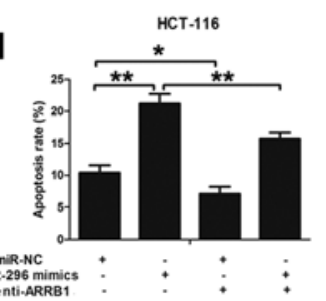

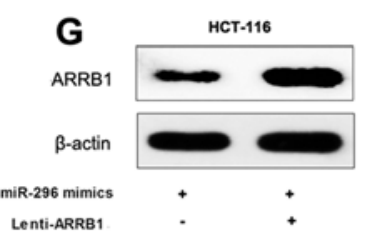
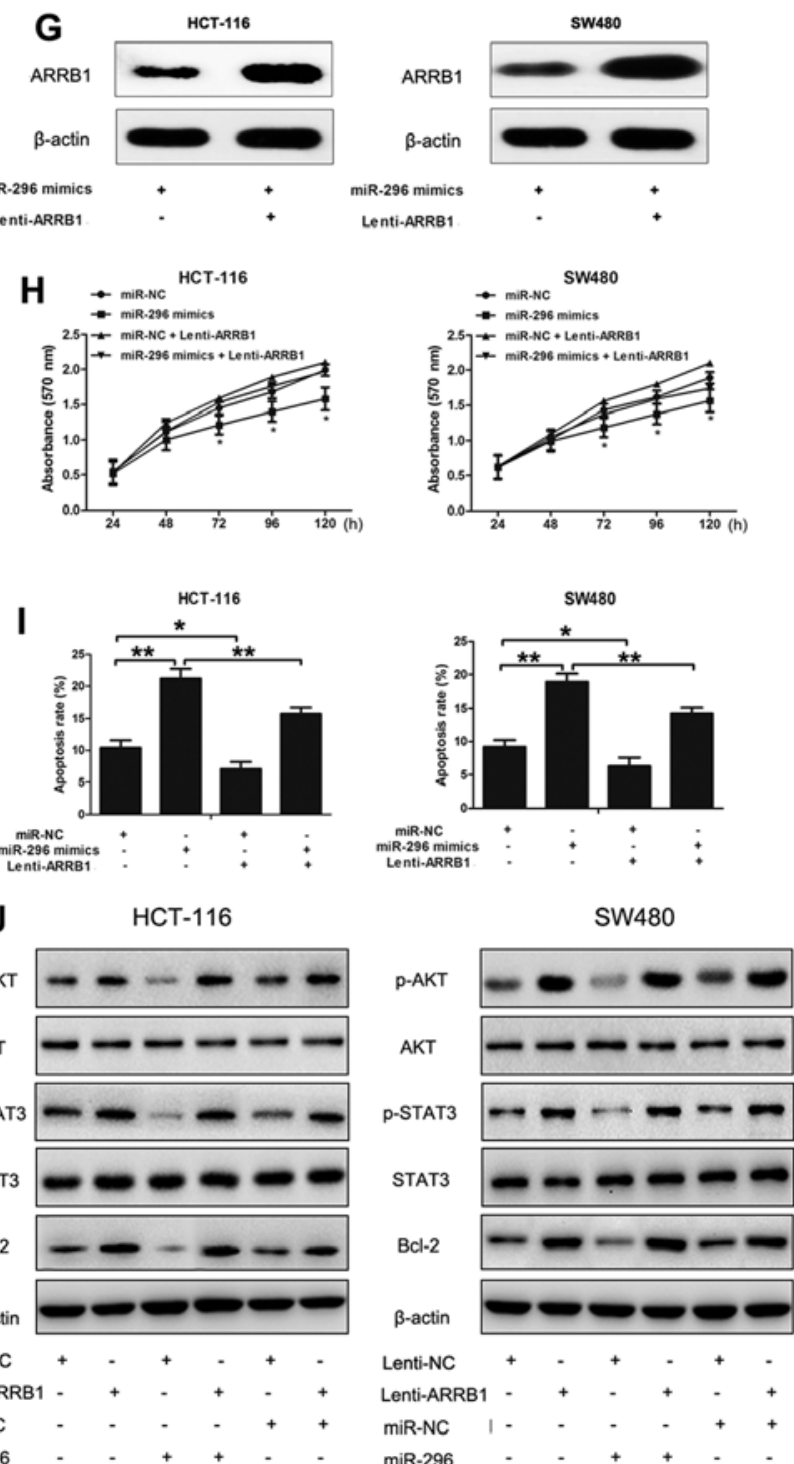

Figure 4. Overexpression of ARRB1 partially rescues miR-296-regulated cell growth and apoptosis in SW480 and HCT-116 cells. (A) mRNA levels of ARRB1 measured in five CRC cell lines by RT-qPCR. "P<0.05 vs. HT-29, SW620 and LoVo cells. (B) Expression of ARRB1 protein in five CRC cell lines measured by western blotting. (C) The mRNA levels of ARRB1 expression were suppressed in SW480 and HCT-116 cells after transfection of ARRB1 siRNA for $72 \mathrm{~h}$, detected by RT-qPCR. "P<0.05 vs. GFP-siRNA-transfected cells. (D) Western blot analysis showing the results of ARRB1 expression in HCT-116 and SW480 cells after ARRB1-siRNA transfection. (E) Effect of ARRB1 underexpression on SW480 and HCT-116 cell proliferation, as measured by MTT assay. * $<0.05$ vs. GFP-siRNA-transfected cells. (F) Effect of ARRB1 underexpression on SW480 and HCT-116 cell apoptosis rates. The number of apoptotic cells was significantly higher in SW480 and HCT-116 cells underexpressing ARRB1, as measured by apoptosis assay. *P<0.05. (G) Western blot analysis of CRC cells co-transfected with miR-296 mimics and Lenti-ARRB1. (H) MTT assay of SW480 and HCT-116 cells co-transfected with miR-296 mimics and Lenti-ARRB1 or the control. " P<0.05 vs. SW480 and HCT-116 cells co-transfected with miR-296 mimics and Lenti-ARRB1. (I) Apoptosis assay of SW480 and HCT-116 cells co-transfected with miR-296 mimics and Lenti-ARRB1 or the control. ${ }^{*} \mathrm{P}<0.05,{ }^{* *} \mathrm{P}<0.01$. (J) ARRB1 overexpression blocks the effect of miR-296 on dephosphorylation of AKT signaling without affecting total AKT and STAT3 expression. Western blot analysis also indicated that miR-296 suppressed Bcl-2 protein expression, via regulation of ARRB1. Western blot analyses were performed in triplicate, and representative results are shown. miR, microRNA; NC, negative control; CRC, colorectal cancer; ARRB1, arrestin $\beta 1$; si, small interfering; GFP, green fluorescent protein; p, phosphorylated; AKT, RAC- $\alpha$ serine/ threonine-protein kinase; STAT3, signal transducer and activator of transcription 3; Bcl-2, B cell lymphoma-2.

that both ARRB1 mRNA and protein expression levels were significantly inhibited by siRNA targeting ARRB1 (Fig. 4C and D). An MTT assay was next used to investigate the contribution of ARRB1 to SW480 and HCT-116 cell proliferation. It was observed that downregulation of ARRB1 led to a significant reduction in survival of SW480 and HCT-116 cells (Fig. 4E). In addition, flow cytometry analysis revealed that SW480 and HCT-116 cells that underexpressed ARRB1 exhibited a significantly increased rate of apoptosis relative to cells expressing the GFP-siRNA vector (Fig. 4F). These results clearly indicated that ARRB1 functions as a critical oncogene through enhancing cell survival and suppressing cell apoptosis in vitro.

miR-296 inhibits cell growth and promotes cell apoptosis through suppressing ARRB1-mediated AKT activation. To figure out whether ARRB1 is a critical mediator in miR296-regulated cell survival and apoptosis, full-length ARRB1 was transfected into SW480 and HCT-116 cells overexpressing miR-296. It was verified that miR-296 mimics significantly 
decreased ARRB1 protein levels and this effect could be in part alleviated by ARRB1 transfection in CRC cells, detected by western blot analysis (Fig. 4G). The MTT and apoptosis assays revealed that upregulation of ARRB1 in SW480 and HCT-116 cells transfected with miR-296 mimics reversed the decreased growth and enhanced apoptosis that was observed in cells transfected only with miR-296 mimics (Fig. 4H and I). These results implied that miR-296 suppresses cell growth and induces cell apoptosis by an ARRB1-dependent mechanism.

The association between miR-296 and ARRB1 in SW480 and HCT-116 cells prompted investigation of the activation of the AKT signaling pathway under altering ARRB1 expression levels. Following enhancement of the expression of ARRB1 in SW480 and HCT-116 cells by using Lenti-ARRB1, it was observed that ARRB1 activated the AKT signaling pathway by increasing the phosphorylation levels of AKT and STAT3 without affecting the total AKT and STAT3 expression. The expression levels of Bcl-2 increased in CRC cells transfected with Lenti-ARRB1. Notably, overexpression of ARRB1 significantly abrogated the decrease in the phosphorylation of AKT and phosphorylation of STAT3 in cells overexpressing miR-296 (Fig. 4J). In addition, ARRB1 rescued cells from the inhibitory effect of miR-296 on Bcl-2 expression (Fig. 4J). These results demonstrated that miR-296-induced apoptosis in CRC may be associated with the inactivation of AKT/STAT3 signaling pathway, which is regulated by ARRB1.

\section{Discussion}

Previous evidence has indicated that miR-296 serves as a tumor suppressor in a variety of malignant cancer cells. Previously, Shivapurkar et al (23) demonstrated that underexpression of miR-296 in serum predicts chemotherapy resistance in patients receiving systemic chemotherapy for metastatic colon cancer. Furthermore, it was reported that miR-296 inhibits CRC metastasis by mediating S100A4, which highlights the potential of miR-296 as an important target against CRC metastasis (24). These results prompted speculation that miR-296 may serve as a tumor suppressor for CRC cells. The present study revealed that miR-296 was underexpressed in $89.28 \%$ of the CRC patients. Kaplan-Meier analysis revealed that lower miR-296 expression was positively associated with poor prognosis. The results identified miR-296 as a promising predictive biomarker, which may aid in the optimization of treatment stratification. Notably, it was demonstrated that SW480 and HCT-116 cells revealed significantly lower expression levels of miR-296 compared with HT-29, SW620, and LoVo cells. It has been reported that miR-296 directly targets protein kinase $\mathrm{C} \alpha$ to suppress focal adhesion kinase-Ras-c-Myc signaling, thus stimulating its own expression in a feedback loop in lung adenocarcinoma (25). Deng et al (26) demonstrated that suppression of MK2 expression downregulates Ras/Braf/Erk/Mek/c-Myc axis and promotes cytoplasmic translocation of c-Myc, which activates miR-296 expression by a feedback loop (26). ATCC information suggests that both SW480 and HCT-116 cells have mutations in the Ras proto-oncogene, while the other three lines (HT-29, SW620, and LoVo cells) have no mutations in the Ras proto-oncogene. Perhaps these altered Ras mutations may contribute to the lower expression level of miR-296. However, this issue requires further studies in the future.
Over the past decades, a wide range of studies have demonstrated that the AKT signaling pathway is associated with multiple cellular functions. Accumulating evidence suggests that the AKT signaling pathway regulates various cellular biological processes including cell apoptosis, proliferation, angiogenesis, and invasion, all of which are involved in CRC development. It is noteworthy that miR-296 targets AKT2 in pancreatic cancer and functions as a potential tumor suppressor (27). Therefore, it is reasonable to speculate that miR-296 may mediate several important signaling pathways, particularly the AKT signaling pathway, and ultimately contributes to tumorigenesis. ARRB1 serves as a ubiquitous, multifunctional scaffolding protein, and it connects the activated receptors with diverse signaling pathways within the cell $(12,17)$. ARRB1 may have different effects on cellular processes in different signaling pathways. The repressed protein ARRB1 indirectly regulates transcription factors involved in DNA damage processing and apoptosis through binding to regulators such as $\mathrm{I} \kappa \mathrm{B} \alpha$ and MDM2 (28). Furthermore, it was reported that ARRB1 mediates prolonged phosphorylation of AKT in glioblastoma cells induced by NK1R activation (29). Given the importance of ARRB1 in the AKT signaling pathway, the present study examined the expression of ARRB1 after miR-296 transfection, to clarify the mechanisms of apoptosis in CRC cells. It was indicated that miR-296 enhanced CRC cell apoptosis by inhibiting ARRB1 expression, a novel downstream target of miR-296, which serves to reduce AKT signaling pathway activity, leading to increased apoptosis. However, the in vivo functions and molecular mechanism of miR-296 and ARRB1 in CRC remain to be fully elucidated, and further studies are required in the future.

Apoptosis is an inborn process that allows the cells to systematically inactivate, destroy their own components and ultimately results in cell death. Bcl-2 is a proto-oncogene that suppresses apoptosis and has been indicated in the pathogenesis of various tumor types including CRC (30). In addition, $\mathrm{CRC}$ with high $\mathrm{Bcl}-2$ expression levels exhibits lower rates of spontaneous apoptosis compared with CRC with low Bcl-2 expression (31). Aberrant activation of the Bcl-2 gene may be an early event in colorectal carcinogenesis that can suppress apoptosis in vivo (31). Interestingly, miR-296 is also suppressed at the premalignant stages of colon carcinogenesis (11). The present study indicated that miR-296 regulates Bcl-2 expression in CRC cells, induced by AKT pathway inactivation. The lack of Bcl-2 activation in miR-296 transfected CRC cells was at least partly responsible for the enhanced cell apoptosis. Moreover, it was demonstrated that overexpression of miR-296 in $\mathrm{CRC}$ resulted in a decrease in Bcl-2 expression, whereas the level of Bax, or PUMA remained unchanged, implying a functional interaction between miR-296 and the Bcl-2-depedent apoptosis pathways. Although there is no direct interaction of miR-296 with Bcl-2, it is reasonable to speculate that miR-296 plays a critical role in the Bcl-2 pathway. Additional studies are essential to fully describe the miR-296-associated Bcl-2 pathway in the future.

In conclusion, the results of the present study indicated that ARRB1 is a direct target of miR-296. In addition, miR-296 resulted in the promotion of cell apoptosis and the suppression of cell growth by regulating the ARRB1-AKT-mediated signaling pathway. The results add to the accumulating evidence 
that miR-296 plays a critical role in CRC tumorigenesis and targeting miR-296 may represent an ideal gene-targeting strategy for CRC treatment.

\section{Acknowledgements}

Not applicable.

\section{Funding}

No funding was received.

\section{Availability of data and materials}

The datasets used and/or analyzed during the current study are available from the corresponding author on reasonable request.

\section{Authors' contributions}

$\mathrm{ZZ}$ and $\mathrm{CC}$ designed and performed the experiments; $\mathrm{YX}, \mathrm{ZZ}$, and $\mathrm{XZ}$ contributed to the data analysis; $\mathrm{CC}$ enrolled patients and measured the RNA levels in the clinical samples; ZZ initiated the study and wrote the manuscript; and all authors have read and approved the final version of the manuscript.

\section{Ethics approval and consent to participate}

The use of human tissues was approved by the Specialty Committee on Ethics of Biomedicine Research (PJ2008012-03), Huizhou Municipal Central Hospital, and patient consent was obtained.

\section{Patient consent for publication}

Written informed consent was obtained.

\section{Competing interests}

The authors declare that they have no competing interests.

\section{References}

1. Wang J, Song YX, Ma B, Wang JJ, Sun JX, Chen XW, Zhao JH, Yang YC and Wang ZN: Regulatory roles of non-coding RNAs in colorectal cancer. Int J Mol Sci 16: 19886-19919, 2015.

2. Konda B, Shum H and Rajdev L: Anti-angiogenic agents in metastatic colorectal cancer. World J Gastrointest Oncol 7: 71-86, 2015

3. Kocarnik JM, Shiovitz S and Phipps AI: Molecular phenotypes of colorectal cancer and potential clinical applications. Gastroenterol Rep (Oxf) 3: 269-276, 2015.

4. Farazi TA, Hoell JI, Morozov P and Tuschl T: MicroRNAs in human cancer. Adv Exp Med Biol 774: 1-20, 2013.

5. Wang L, Bo X, Zheng Q, Xiao X, Wu L and Li B: miR-296 inhibits proliferation and induces apoptosis by targeting FGFR1 in human hepatocellular carcinoma. FEBS Lett 590: 4252-4262, 2016.

6. Dinami R, Buemi V, Sestito R, Zappone A, Ciani Y, Mano M, Petti E, Sacconi A, Blandino G, Giacca M, et al: Epigenetic silencing of miR-296 and miR-512 ensures hTERT dependent apoptosis protection and telomere maintenance in basal-type breast cancer cells. Oncotarget 8: 95674-95691, 2017.

7. Lee KH, Lin FC, Hsu TI, Lin JT, Guo JH, Tsai CH, Lee YC, Lee YC, Chen CL, Hsiao M, et al: MicroRNA-296-5p (miR-296-5p) functions as a tumor suppressor in prostate cancer by directly targeting Pin1. Biochim Biophys Acta 1843: 2055-2066, 2014.
8. Yan W, Chen J, Chen Z and Chen H: Deregulated miR-296/ S100A4 axis promotes tumor invasion by inducing epithelialmesenchymal transition in human ovarian cancer. Am J Cancer Res 6: 260-269, 2016.

9. Luo W, Lin Y, Meng S, Guo Y, Zhang J and Zhang W: miRNA296-3p modulates chemosensitivity of lung cancer cells by targeting CX3CR1. Am J Transl Res 8: 1848-1856, 2016.

10. Savi F, Forno I, Faversani A, Luciani A, Caldiera S, Gatti S, Foa P, Ricca D, Bulfamante G, Vaira V, et al: miR-296/Scribble axis is deregulated in human breast cancer and miR-296 restoration reduces tumour growth in vivo. Clin Sci (Lond) 127: 233-242, 2014.

11. Kunte DP, DelaCruz M, Wali RK, Menon A, Du H, Stypula Y, Patel A, Backman V and Roy HK: Dysregulation of microRNAs in colonic field carcinogenesis: Implications for screening. PLoS One 7: e45591, 2012.

12. Witherow DS, Garrison TR, Miller WE and Lefkowitz RJ: betaArrestin inhibits NF-kappaB activity by means of its interaction with the NF-kappaB inhibitor IkappaBalpha. Proc Natl Acad Sci USA 101: 8603-8607, 2004

13. Miele E, Po A, Begalli F, Antonucci L, Mastronuzzi A, Marras CE, Carai A, Cucchi D, Abballe L, Besharat ZM, et al: $\beta$-arrestin1-mediated acetylation of Gli1 regulates Hedgehog/Gli signaling and modulates self-renewal of SHH medulloblastoma cancer stem cells. BMC Cancer 17: 488, 2017.

14. Kraemer A, Barjaktarovic Z, Sarioglu H, Winkler K, EckardtSchupp F, Tapio S, Atkinson MJ and Moertl S: Cell survival following radiation exposure requires miR-525-3p mediated suppression of ARRB1 and TXN1. PLoS One 8: e77484, 2013.

15. Kang J, Shi Y, Xiang B, Qu B, Su W, Zhu M, Zhang M, Bao G, Wang F, Zhang X, et al: A nuclear function of beta-arrestin1 in GPCR signaling: Regulation of histone acetylation and gene transcription. Cell 123: 833-847, 2005.

16. Parathath SR, Mainwaring LA, Fernandez-L A, Guldal CG, Nahlé $\mathrm{Z}$ and Kenney AM: $\beta$-Arrestin-1 links mitogenic sonic hedgehog signaling to the cell cycle exit machinery in neural precursors. Cell Cycle 9: 4013-4024, 2010.

17. Yang Y, Guo Y, Tan S, Ke B, Tao J, Liu H, Jiang J, Chen J, Chen G and $\mathrm{Wu} \mathrm{B}$ : $\beta$-Arrestin1 enhances hepatocellular carcinogenesis through inflammation-mediated Akt signalling. Nat Commun 6: $7369,2015$.

18. Zecchini V, Madhu B, Russell R, Pértega-Gomes N, Warren A, Gaude E, Borlido J, Stark R, Ireland-Zecchini H, Rao R, et al: Nuclear ARRB1 induces pseudohypoxia and cellular metabolism reprogramming in prostate cancer. EMBO J 33: 1365-1382, 2014.

19. Livak KJ and Schmittgen TD: Analysis of relative gene expression data using real-time quantitative PCR and the 2(-Delta Delta C(T)) Method. Methods 25: 402-408, 2001.

20. Ma H, Wang L, Zhang T, Shen H and Du J: Loss of $\beta$-arrestin1 expression predicts unfavorable prognosis for non-small cell lung cancer patients. Tumour Biol 37: 1341-1347, 2016.

21. Hong L, Han Y, Zhang H, Li M, Gong T, Sun L, Wu K, Zhao Q and Fan D: The prognostic and chemotherapeutic value of miR-296 in esophageal squamous cell carcinoma. Ann Surg 251: 1056-1063, 2010.

22. Wang Q, Qian J, Wang J, Luo C, Chen J, Hu G and Lu Y: Knockdown of RLIP76 expression by RNA interference inhibits invasion, induces cell cycle arrest, and increases chemosensitivity to the anticancer drug temozolomide in glioma cells. J Neurooncol 112: 73-82, 2013

23. Shivapurkar N, Mikhail S, Navarro R, Bai W, Marshall J, Hwang J, Pishvaian M, Wellstein A and He AR: Decrease in blood miR-296 predicts chemotherapy resistance and poor clinical outcome in patients receiving systemic chemotherapy for metastatic colon cancer. Int J Colorectal Dis 28: 887, 2013.

24. He Z, Yu L, Luo S, Li M, Li J, Li Q, Sun Y and Wang C: miR-296 inhibits the metastasis and epithelial-mesenchymal transition of colorectal cancer by targeting S100A4. BMC Cancer 17: 140, 2017.

25. Fu Q, Song X, Liu Z, Deng X, Luo R, Ge C, Li R, Li Z, Zhao M, Chen Y, et al: miRomics and proteomics reveal a miR-296-3p/ PRKCA/FAK/Ras/c-Myc feedback loop modulated by HDGF/ DDX5/ $\beta$-catenin complex in lung adenocarcinoma. Clin Cancer Res 23: 6336-6350, 2017.

26. Deng X, Liu Z, Liu X, Fu Q, Deng T, Lu J, Liu Y, Liang Z, Jiang Q, Cheng C et al: miR-296-3p negatively regulated by nicotine stimulates cytoplasmic translocation of c-Myc via MK2 to suppress chemotherapy resistance. Mol Ther 26: 1066-1081, 2018. 
27. Li H, Li J, Shi B and Chen F: MicroRNA-296 targets AKT2 in pancreatic cancer and functions as a potential tumor suppressor. Mol Med Rep 16: 466-472, 2017.

28. Ma L and Pei G: Beta-arrestin signaling and regulation of transcription. J Cell Sci 120: 213-218, 2007.

29. Zhang YX, Li XF, Yuan GQ, Hu H, Song XY, Li JY, Miao XK, Zhou TX, Yang WL, Zhang XW, et al: $\beta$-Arrestin 1 has an essential role in neurokinin-1 receptor-mediated glioblastoma cell proliferation and G2/M phase transition. J Biol Chem 292: 8933-8947, 2017.
30. Guzińska-Ustymowicz K, Pryczynicz A, Kemona A and Czyzewska J: Correlation between proliferation markers: PCNA, $\mathrm{Ki}-67, \mathrm{MCM}-2$ and antiapoptotic protein Bcl-2 in colorectal cancer. Anticancer Res 29: 3049-3052, 2009.

31. Sinicrope FA, Ruan SB, Cleary KR, Stephens LC, Lee JJ and Levin B: bcl-2 and p53 oncoprotein expression during colorectal tumorigenesis. Cancer Res 55: 237-241, 1995. 